\title{
Assessment of marine environment by ABC (abundance biomass comparison) analysis-A case study on western offshore area of Arabian Sea
}

\author{
G L Das *, Pramod Kumar and Atul Garg \\ Environment Division, IPSHEM- ONGC Goa India.
}

World Journal of Advanced Engineering Technology and Sciences, 2021, 02(01), 069-081

Publication history: Received on 06 February 2021; revised on 15 March 2021; accepted on 17 March 2021

Article DOI: https://doi.org/10.30574/wjaets.2021.2.1.0027

\begin{abstract}
Assessment of the variations in marine ecosystem can be effectively monitored using benthic fauna because pollutants from any source will ultimately end in the seabed. The benthic communities play an important role in the transfer of materials from primary production through detrital pool into higher tropic levels, including commercially exploitable fish. Majority of the benthic fauna are sedentary and sessile in nature and cannot avoid any environmental perturbation, hence are considered sensitive indicator of change in the environment caused by natural and anthropogenic disturbances. Based on the analysis of abundance and biomass of benthic communities, one very important and effective study in analyzing marine environment i.e. ABC (Abundance-Biomass Comparison) has been adopted in this paper.
\end{abstract}

Keywords: E\&P activities; Mumbai High; ABC curve

\section{Introduction}

ABC curves have a theoretical background in classical evolutionary theory of $r$ - and k-selection. In undisturbed states, the community is supposed to be dominated by k-selected species (slow-growing, large, late maturing), and the biomass curve lies above the abundance curve. With increasing disturbance, slow-growing species cannot cope, and the system is increasingly dominated by r-selected species (fast-growing, small, opportunistic), and the biomass curve will be below the abundance curve. The difference between the two curves is given by the W-statistic, which represents the area between them. The ABC method takes into account the number of species included in the analysis. [1] [2] An advantage of the method is that an appropriate data set for any area or time should allow the status of the community to be evaluated without the need for a spatial or a temporal control against which to compare the index obtained, because the biomass is compared with the abundance for the same time and place. ${ }^{[1] ~[2] ~}$

This paper aims to explore the validity and possibility of this method by considering the abundance and biomass data around the 21 ONGC offshore installations and drilling rigs (R-12A, HRA, NLM, BHS, NA, NQ, ICP, SHP, BLQ, B-193, B192, TPP-TCPP, WO-16, Sagar Gaurav B-155-B, B-127, Great drill Chaaru B-127N-C, B-48, GS-0SN-2004/1, GK-28/42, TPPTCPP and reference points around Mumbai High, South Basin, Neelam Basin, Heera Basin during 2020-21.

\section{Experimental procedure}

\subsection{Study area}

The study area extends geographically from $18^{\circ} 16^{\prime} 20.32^{\prime \prime} \mathrm{N}$ to $22^{\circ} 37^{\prime} 15.27^{\prime \prime} \mathrm{N}, 71^{\circ} 1^{\prime} 40.59^{\prime \prime} \mathrm{E}$ to $72^{\circ} 30^{\prime} 0.00^{\prime \prime} \mathrm{E}$ covering oil and gas fields of ONGC in the Western continental shelf. The cruise route (Fig: 1) and coordinates/water depth of the

\footnotetext{
* Corresponding author: G L Das

GM (Chem)-Head-Environment, IPSHEM- ONGC Goa India.
}

Copyright (C) 2021 Author(s) retain the copyright of this article. This article is published under the terms of the Creative Commons Attribution Liscense 4.0. 
installations are given bellow.

Table 1(a) Details of sampling locations - platforms \& rigs

\begin{tabular}{|c|c|c|c|}
\hline Sr. No. & Platform & Latitude & Longitude \\
\hline 1 & R-12A (Ratnagiri) & $18^{\circ} 16^{\prime} 20.32^{\prime \prime} \mathrm{N}$ & $72^{\circ} 22^{\prime} 48.52^{\prime \prime} \mathrm{E}$ \\
\hline 2 & HRA (Heera) & $18^{\circ} 34^{\prime} 37.20^{\prime \prime} \mathrm{N}$ & $72^{\circ} 14^{\prime} 27.00^{\prime \prime} \mathrm{E}$ \\
\hline 3 & NLM (Neelam) & $18^{\circ} 42^{\prime} 16.80^{\prime \prime} \mathrm{N}$ & $72^{\circ} 20^{\prime} 17.40 " \mathrm{E}$ \\
\hline 4 & B-193 & $18^{\circ} 55^{\prime} 35.68^{\prime \prime} \mathrm{N}$ & $72^{\circ} 2 ' 29.14 " \mathrm{E}$ \\
\hline 5 & BLQ & $19^{\circ} 12^{\prime} 16.80^{\prime \prime} \mathrm{N}$ & $72^{\circ} 6{ }^{\prime} 27.00^{\prime \prime} \mathrm{E}$ \\
\hline 6 & W016 & $18^{\circ} 58^{\prime} 16.34^{\prime \prime} \mathrm{N}$ & $71^{\circ} 37^{\prime} 18.03^{\prime \prime E}$ \\
\hline 7 & NBP Field & $18^{\circ} 36^{\prime} 9.93^{\prime \prime} \mathrm{N}$ & $71^{\circ} 1^{\prime} 40.59 " \mathrm{E}$ \\
\hline 8 & B-192-5 & $19^{\circ} 10^{\prime} 37.02^{\prime \prime} \mathrm{N}$ & $70^{\circ} 57^{\prime} 43.02^{\prime \prime} \mathrm{E}$ \\
\hline 9 & ICP & $19^{\circ} 20^{\prime} 30.00^{\prime \prime} \mathrm{N}$ & $71^{\circ} 18^{\prime} 0.00^{\prime \prime E}$ \\
\hline 10 & BHS & $19^{\circ} 22^{\prime} 12.00^{\prime \prime} \mathrm{N}$ & $71^{\circ} 21^{\prime} 24.00^{\prime \prime} \mathrm{E}$ \\
\hline 11 & SHP & $19^{\circ} 16^{\prime} 24.00^{\prime \prime} \mathrm{N}$ & $71^{\circ} 25^{\prime} 37.20^{\prime \prime} \mathrm{E}$ \\
\hline 12 & SCA & $19^{\circ} 25^{\prime} 2.25^{\prime \prime} \mathrm{N}$ & $71^{\circ} 23^{\prime} 22.08^{\prime \prime E}$ \\
\hline 13 & NA & $19^{\circ} 34^{\prime} 15.00^{\prime \prime} \mathrm{N}$ & $71^{\circ} 21^{\prime} 32.40^{\prime \prime} \mathrm{E}$ \\
\hline 14 & NQ & $19^{\circ} 34^{\prime} 15.00^{\prime \prime} \mathrm{N}$ & $71^{\circ} 21^{\prime} 32.40^{\prime \prime} \mathrm{E}$ \\
\hline 15 & Sagar Gaurav B-155-B & $19^{\circ} 25^{\prime} 23.57^{\prime \prime} \mathrm{N}$ & $71^{\circ} 30^{\prime} 55.42^{\prime \prime} \mathrm{E}$ \\
\hline 16 & $\mathrm{~B}-127$ & $19^{\circ} 28^{\prime} 41.99^{\prime \prime} \mathrm{N}$ & 714'39.99"E \\
\hline 17 & Great drill Chaaru B-127N-C & $19^{\circ} 36^{\prime} 23.07^{\prime \prime} \mathrm{N}$ & $71^{\circ} 47^{\prime} 48.87^{\prime \prime} \mathrm{E}$ \\
\hline 18 & B-48 & $19^{\circ} 37^{\prime} 32.77^{\prime \prime} \mathrm{N}$ & $71^{\circ} 2^{\prime} 23.97^{\prime \prime E}$ \\
\hline 19 & GS-OSN-2004/1 & $21^{\circ} 37^{\prime} 23.26^{\prime \prime} \mathrm{N}$ & $68^{\circ} 26^{\prime} 32.99^{\prime \prime} \mathrm{E}$ \\
\hline 20 & GK-28/42 & $22^{\circ} 37^{\prime} 15.27^{\prime \prime N}$ & $68^{\circ} 27^{\prime} 08.98^{\prime \prime} \mathrm{E}$ \\
\hline 21 & ТPP-TCPP & $20^{\circ} 36^{\prime} 3.20^{\prime \prime} \mathrm{N}$ & $72^{\circ} 2^{\prime} 10.20^{\prime \prime E}$ \\
\hline
\end{tabular}

Table 1(b) Details of sampling locations - reference points

\begin{tabular}{|l|l|l|l|}
\hline Reference Locations & Sampling Location Number & Latitude & Longitude \\
\hline \multirow{5}{*}{ Mumbai High Reference Point } & MHRF-1 & $19^{\circ} 48^{\prime} 55.00^{\prime \prime} \mathrm{N}$ & $71^{\circ} 4^{\prime} 30.00^{\prime \prime} \mathrm{E}$ \\
\cline { 2 - 4 } & MHRF-2 & $19^{\circ} 34^{\prime} 0.00^{\prime \prime} \mathrm{N}$ & $71^{\circ} 4^{\prime} 30.00^{\prime \prime} \mathrm{E}$ \\
\cline { 2 - 4 } & MHRF-3 & $19^{\circ} 18^{\prime} 0.00^{\prime \prime} \mathrm{N}$ & $71^{\circ} 4^{\prime} 30.00^{\prime \prime} \mathrm{E}$ \\
\cline { 2 - 4 } & MHRF-4 & $19^{\circ} 4^{\prime} 20.00^{\prime \prime} \mathrm{N}$ & $71^{\circ} 4^{\prime} 30.00^{\prime \prime} \mathrm{E}$ \\
\cline { 2 - 4 } & MHRF-5 & $19^{\circ} 48^{\prime} 55.00^{\prime \prime} \mathrm{N}$ & $71^{\circ} 16^{\prime} 00.00^{\prime \prime} \mathrm{E}$ \\
\cline { 2 - 4 } & MHRF-6 & $19^{\circ} 48^{\prime} 55.00^{\prime \prime} \mathrm{N}$ & $71^{\circ} 30^{\prime} 0.00^{\prime \prime} \mathrm{E}$ \\
\cline { 2 - 4 } & MHRF-7 & $19^{\circ} 48^{\prime} 55.00^{\prime \prime} \mathrm{N}$ & $71^{\circ} 41^{\prime} 30.00^{\prime \prime} \mathrm{E}$ \\
\cline { 2 - 4 } & MHRF-8 & $19^{\circ} 36^{\prime} 0.01^{\prime \prime} \mathrm{N}$ & $71^{\circ} 41^{\prime} 30.00^{\prime \prime} \mathrm{E}$ \\
\cline { 2 - 4 } & MHRF-9 & $19^{\circ} 22^{\prime} 0.03^{\prime \prime} \mathrm{N}$ & $71^{\circ} 41^{\prime} 30.00^{\prime \prime} \mathrm{E}$ \\
\cline { 2 - 4 } & MHRF-10 & $19^{\circ} 4^{\prime} 20.01^{\prime \prime} \mathrm{N}$ & $71^{\circ} 41^{\prime} 30.00^{\prime \prime} \mathrm{E}$ \\
\cline { 2 - 4 } & MHRF-11 & $19^{\circ} 4^{\prime} 20.00^{\prime \prime} \mathrm{N}$ & $71^{\circ} 32^{\prime} 0.00^{\prime \prime} \mathrm{E}$ \\
\cline { 2 - 4 } South Basin Reference Point & $19^{\circ} 4^{\prime} 20.00^{\prime \prime} \mathrm{N}$ & $71^{\circ} 18^{\prime} 0.03^{\prime \prime} \mathrm{E}$ \\
\hline & MHRF-12 & $19^{\circ} 26^{\prime} 0.00^{\prime \prime} \mathrm{N}$ & $71^{\circ} 54^{\prime} 30.00^{\prime \prime} \mathrm{E}$ \\
\hline & S-Basin-1 & $19^{\circ} 26^{\prime} 0.00^{\prime \prime} \mathrm{N}$ & $71^{\circ} 54^{\prime} 30.00^{\prime \prime} \mathrm{E}$ \\
\cline { 2 - 4 } & S-Basin-2 & $18^{\circ} 49^{\prime} 0.00^{\prime \prime} \mathrm{N}$ & $71^{\circ} 54^{\prime} 30.00^{\prime \prime} \mathrm{E}$ \\
\cline { 2 - 4 } & S-Basin-3 & $18^{\circ} 49^{\prime} 0.00^{\prime \prime} \mathrm{N}$ & $72^{\circ} 16^{\prime} 00.00^{\prime \prime} \mathrm{E}$ \\
\cline { 2 - 4 } & S-Basin-4 & &
\end{tabular}




\begin{tabular}{|l|l|l|l|}
\hline Reference Locations & Sampling Location Number & Latitude & Longitude \\
\hline \multirow{4}{*}{ Neelam Basin Reference Point } & Satellite N-1 & $18^{\circ} 56^{\prime} 50.00 " \mathrm{~N}$ & $72^{\circ} 11^{\prime} 20.00 " \mathrm{E}$ \\
\cline { 2 - 4 } & Satellite N-2 & $18^{\circ} 31^{\prime} 20.00^{\prime \prime} \mathrm{N}$ & $72^{\circ} 11^{\prime} 20.00 " \mathrm{E}$ \\
\cline { 2 - 4 } & Satellite N-3 & $18^{\circ} 31^{\prime} 20.00^{\prime \prime} \mathrm{N}$ & $72^{\circ} 30^{\prime} 0.00^{\prime \prime} \mathrm{E}$ \\
\cline { 2 - 4 } & Satellite N-4 & $18^{\circ} 56^{\prime} 50.00^{\prime \prime} \mathrm{N}$ & $72^{\circ} 30^{\prime} 00.00^{\prime \prime} \mathrm{E}$ \\
\hline \multirow{5}{*}{ Heera Basin Reference Point } & Satellite H-1 & $18^{\circ} 47^{\prime} 45.00^{\prime \prime} \mathrm{N}$ & $72^{\circ} 55^{\prime} 20.04^{\prime \prime} \mathrm{E}$ \\
\cline { 2 - 4 } & Satellite H-2 & $18^{\circ} 20^{\prime} 0.00^{\prime \prime} \mathrm{N}$ & $72^{\circ} 5{ }^{\prime} 20.00^{\prime \prime} \mathrm{E}$ \\
\cline { 2 - 4 } & Satellite H-3 & $18^{\circ} 20^{\prime} 0.00^{\prime \prime} \mathrm{N}$ & $72^{\circ} 26^{\prime} 0.00^{\prime \prime} \mathrm{E}$ \\
\cline { 2 - 4 } & Satellite H-4 & $18^{\circ} 47^{\prime} 45.00^{\prime \prime} \mathrm{N}$ & $72^{\circ} 26^{\prime} 0.00^{\prime \prime} \mathrm{E}$ \\
\hline
\end{tabular}

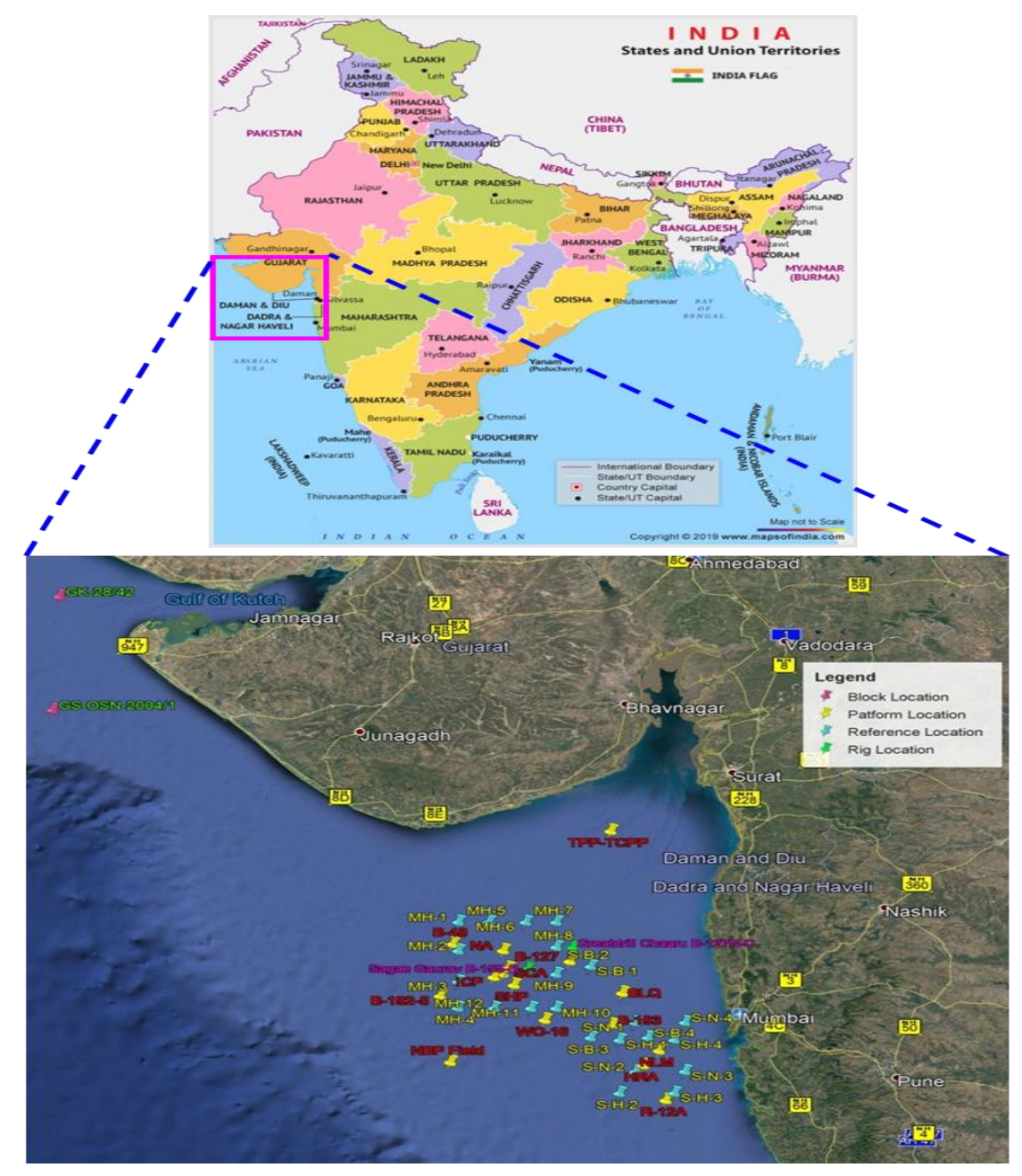

Figure 1 Index map

\subsection{Field sampling}

Based on OSPAR Guideline ${ }^{[3]}$ (Fig: 2) and keeping in view the pipeline network in the vicinity of the platforms in addition to sea state and manoeuvrability of the vessel around the installations.

Samples are collected at stations scattered in circles of $250 \mathrm{~m}, 500 \mathrm{~m}, 1000 \mathrm{~m}, 2000 \mathrm{~m}$ and $4000 \mathrm{~m}$ surrounding each installation. Reference samples were collected beyond $10 \mathrm{kms}$ from the installations. Sea bottom dwelling organisms or benthos are collected at each stations by lowering a Van Veen Grab having penetration depth of $10 \mathrm{cms}$ and variable surface coverage of $587.5 \mathrm{~cm} 2$ ( small grab) and $1035 \mathrm{~cm} 2$ ( large grab). 


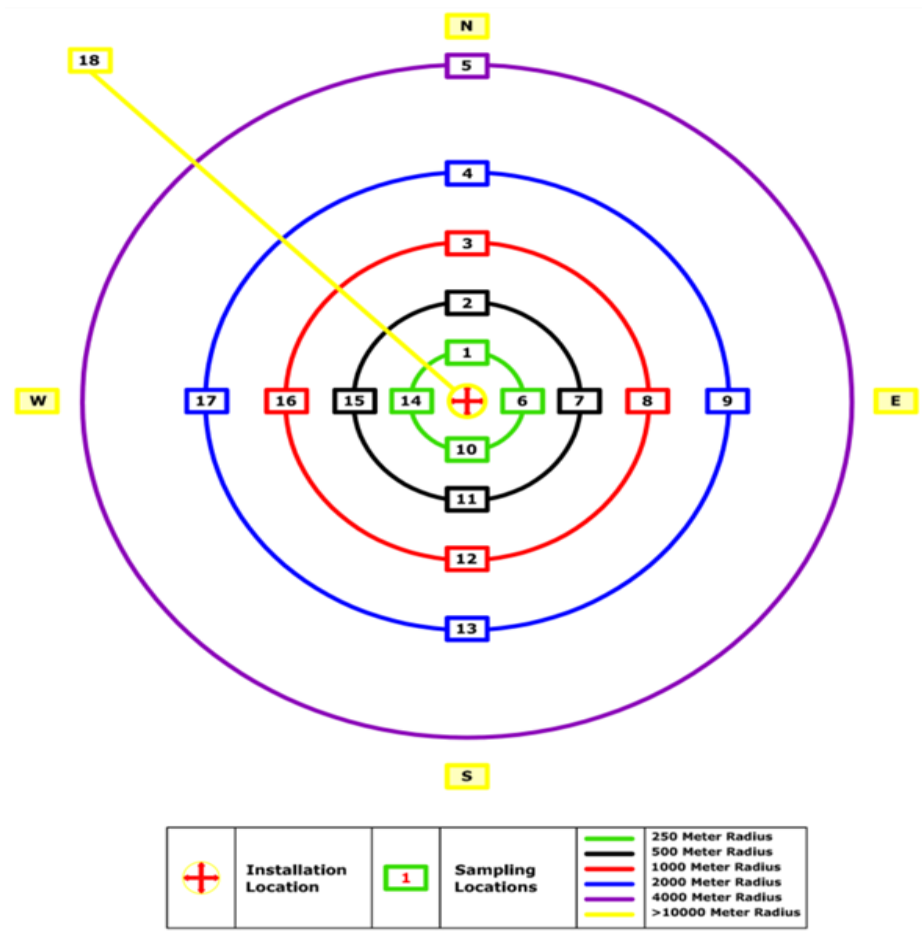

Figure 2 OSPAR Commission Sampling Strategy

\subsection{Laboratory analysis}

All the macro faunal samples were processed on board after $48 \mathrm{hrs}$. of collection using 500 micron stainless steel mesh screen in filtered seawater and material retained on sieve mesh were fixed in 5\% formalin Rose Bengal. In biological laboratory, all the fauna was stored, identified up to the lower possible level under the Microscope. Biomass (wet weight) was measured by blotting the sample on a blotting paper and weight was taken by direct weighing on balance. The biomass was calculated in $\mathrm{g} / \mathrm{m} 2$.

\subsection{Data analysis}

For construction of ABC curve, Primer (version 6), a software made for statistical analysis for ecological study, was used. Abundance i.e. mean density of different macro benthic species around each stations of an installation are counted as numbers/ meter 2 and the total biomass of different stations are measured as gm. /m2 as listed tables (Table 1 \& Table 2). Now, the tables of abundance and biomass of different stations of a particular installation are incorporated in the software (primer V6). After running the software as "cumulative dominance curve" one ABC curve is formed where in $\mathrm{y}$ - axis we will get "cumulative dominance \%" and in $\mathrm{x}$-axis "species rank" will appear. The difference between the two curves is given by the W-statistic, which represents the area between them.

\section{Results and discussion}

- In undisturbed communities the biomass is dominated by one or a few large organisms, leading to an elevated biomass curve, each of these groups are represented by rather few individuals so they do not dominate the abundance curve, which shows a typical diverse equitable distribution.

- Under moderate pollution or disturbances the large competitive dominants are eliminated and the inequality in size between the numerical and biomass and biomass dominates is reduced so that the biomass and abundance curves are closely coincident and may cross each other one or more time (Clarke and Warwick, 2001)

- As pollution become more severe, benthic communities become increasingly dominated by one or a few opportunistic group, which whilst they dominate the numbers do not dominate the biomass, because they are very small bodied, Hence the abundance curve lies above the biomass curve throughout its length(Clarke and Warwick, 2001). 
Table 2 Abundance of Macro Fauna at various stations for different installations (in No/M2)

\begin{tabular}{|c|c|c|c|c|c|c|c|c|c|c|c|c|c|c|c|c|c|c|}
\hline \multirow[t]{2}{*}{ Installations } & \multicolumn{18}{|c|}{ STATIONS } \\
\hline & 1 & 2 & 3 & 4 & 5 & 6 & 7 & 8 & 9 & 10 & 11 & 12 & 13 & 14 & 15 & 16 & 17 & 18 \\
\hline Heera & 310 & 310 & 350 & 280 & 300 & 300 & 330 & 370 & 340 & 330 & 340 & 370 & 330 & 440 & 290 & 320 & 330 & 290 \\
\hline Neelam & 320 & 310 & 250 & 290 & 400 & 300 & 430 & 310 & 300 & 380 & 360 & 420 & 340 & 410 & 380 & 410 & 390 & 430 \\
\hline BLQ & 420 & 440 & 620 & 400 & 430 & 420 & 420 & 440 & 550 & 380 & 450 & 490 & 540 & 440 & 540 & 440 & 530 & 500 \\
\hline $\mathrm{R}-12 \mathrm{~A}$ & 250 & 240 & 260 & 280 & 270 & 220 & 240 & 280 & 360 & 290 & 330 & 250 & 190 & 270 & 240 & 270 & 260 & 330 \\
\hline B-193A & 290 & 240 & 280 & 220 & 320 & 360 & 330 & 290 & 350 & 370 & 350 & 270 & 300 & 350 & 280 & 240 & 250 & 330 \\
\hline WO-16 & 330 & 310 & 300 & 240 & 280 & 300 & 300 & 280 & 350 & 270 & 290 & 310 & 330 & 290 & 280 & 320 & 270 & 320 \\
\hline NBP Field & 360 & 360 & 350 & 340 & 400 & 330 & 290 & 270 & 390 & 340 & 300 & 180 & 290 & 280 & 310 & 320 & 320 & 390 \\
\hline NA & 250 & 280 & 260 & 250 & 230 & 360 & 300 & 320 & 420 & 370 & 360 & 340 & 250 & 310 & 340 & 410 & 260 & 310 \\
\hline NQ & 320 & 200 & 230 & 360 & 230 & 300 & 280 & 290 & 240 & 380 & 220 & 250 & 320 & 220 & 280 & 240 & 230 & 330 \\
\hline SCA & 190 & 180 & 210 & 270 & 200 & 240 & 220 & 280 & 240 & 280 & 230 & 310 & 270 & 250 & 200 & 180 & 270 & 280 \\
\hline SHP & 270 & 290 & 340 & 290 & 360 & 280 & 400 & 250 & 330 & 380 & 380 & 330 & 440 & 430 & 290 & 410 & 260 & 320 \\
\hline ICP & 280 & 280 & 410 & 300 & 370 & 330 & 240 & 410 & 360 & 300 & 450 & 270 & 330 & 360 & 350 & 360 & 370 & 240 \\
\hline BHS & 230 & 380 & 350 & 470 & 330 & 330 & 370 & 370 & 430 & 330 & 310 & 390 & 510 & 460 & 410 & 360 & 340 & 450 \\
\hline B-155-B & 270 & 330 & 290 & 460 & 410 & 360 & 440 & 350 & 280 & 320 & 330 & 400 & 320 & 310 & 360 & 310 & 330 & 300 \\
\hline B-127 & 310 & 370 & 220 & 300 & 380 & 320 & 340 & 290 & 290 & 340 & 380 & 380 & 340 & 340 & 350 & 360 & 340 & 350 \\
\hline B-192-5 & 320 & 330 & 310 & 190 & 290 & 300 & 270 & 220 & 340 & 250 & 380 & 290 & 310 & 260 & 390 & 250 & 310 & 350 \\
\hline B-127N-C & 310 & 310 & 280 & 400 & 470 & 350 & 430 & 320 & 350 & 390 & 360 & 360 & 290 & 310 & 360 & 340 & 340 & 360 \\
\hline B-48 & 140 & 270 & 160 & 160 & 170 & 240 & 250 & 210 & 420 & 280 & 230 & 250 & 290 & 240 & 200 & 230 & 250 & 300 \\
\hline GS-OSN-2004/1 & 280 & 310 & 210 & 310 & 280 & 430 & 270 & 290 & 260 & 200 & 200 & 310 & 190 & 420 & 220 & 190 & 200 & 390 \\
\hline GK-28/42 & 330 & 320 & 290 & 370 & 480 & 360 & 290 & 390 & 310 & 390 & 490 & 330 & 310 & 310 & 350 & 300 & 420 & 320 \\
\hline ТPP-ТCPP & 180 & 250 & 260 & 260 & 170 & 220 & 160 & 170 & 260 & 180 & 170 & 380 & 200 & 250 & 230 & 280 & 230 & 280 \\
\hline MHRF & 430 & 410 & 360 & 440 & 370 & 260 & 510 & 330 & 420 & 410 & 390 & 380 & & & & & & \\
\hline S-BASIN & 350 & 300 & 310 & 240 & & & & & & & & & & & & & & \\
\hline SATELLITE & 290 & 400 & 300 & 340 & 310 & 340 & 240 & 330 & 290 & & & & & & & & & \\
\hline
\end{tabular}


Table 3 Biomass of Macro Fauna at various stations around different Installations (gm/M2)

\begin{tabular}{|c|c|c|c|c|c|c|c|c|c|c|c|c|c|c|c|c|c|c|}
\hline \multirow[t]{2}{*}{ Installations } & \multicolumn{18}{|c|}{ STATIONS } \\
\hline & 1 & 2 & 3 & 4 & 5 & 6 & 7 & 8 & 9 & 10 & 11 & 12 & 13 & 14 & 15 & 16 & 17 & 18 \\
\hline Heera & 4.52 & 3.95 & 3.47 & 2.89 & 3.21 & 3.19 & 3.58 & 3.89 & 4.1 & 3.14 & 3.29 & 3.78 & 3.19 & 6.21 & 4.22 & 3.44 & 3.56 & 4.12 \\
\hline Neelam & 3.24 & 3.14 & 2.98 & 3.14 & 4.12 & 3.45 & 5.22 & 3.19 & 2.98 & 3.59 & 2.87 & 5.04 & 3.25 & 3.68 & 3.74 & 3.49 & 2.99 & 3.48 \\
\hline BLQ & 3.58 & 4.12 & 6.77 & 4.66 & 3.98 & 3.47 & 3.45 & 3.68 & 5.34 & 3.19 & 4.15 & 5.62 & 5.11 & 4.1 & 4.87 & 3.66 & 6.23 & 4.77 \\
\hline $\mathrm{R} 12 \mathrm{~A}$ & 3.01 & 3.65 & 2.84 & 4.12 & 3.84 & 3.14 & 3.12 & 3.58 & 3.78 & 4.1 & 5.12 & 3.14 & 2.98 & 3.74 & 3.59 & 2.84 & 2.95 & 5.22 \\
\hline B193A & 3.45 & 2.88 & 3.86 & 2.96 & 3.02 & 4,11 & 3.22 & 2.48 & 3.56 & 3.15 & 4.55 & 2.78 & 3.14 & 3.24 & 3.41 & 2.91 & 2.86 & 2.78 \\
\hline W0-16 & 3.48 & 3.41 & 2.98 & 2.48 & 2.99 & 3.48 & 2.58 & 3.47 & 5.23 & 3.47 & 2.69 & 3.25 & 4.44 & 3.26 & 2.98 & 4.45 & 3.58 & 4.15 \\
\hline NBP Field & 3.48 & 4.12 & 3.98 & 3.47 & 5.34 & 3.45 & 2.48 & 2.69 & 3.44 & 3.16 & 2.98 & 2.48 & 3.25 & 3.14 & 3.68 & 3.17 & 3.58 & 4.22 \\
\hline NA & 3.57 & 3.96 & 3.61 & 3.48 & 3.42 & 4.12 & 4.01 & 3.65 & 5.12 & 4.24 & 4.35 & 4.22 & 3.59 & 4.15 & 4.36 & 5.01 & 3.74 & 3.15 \\
\hline $\mathrm{NQ}$ & 4.21 & 2.56 & 2.84 & 4.69 & 2.89 & 2.99 & 2.48 & 4.01 & 3.69 & 4.65 & 2.84 & 2.96 & 3.1 & 2.84 & 3.69 & 2.81 & 3.71 & 3.24 \\
\hline SCA & 2.69 & 2.54 & 3.14 & 3.57 & 3.26 & 3.47 & 3.47 & 3.68 & 3.48 & 3.69 & 3.54 & 3.15 & 3.56 & 3.64 & 3.29 & 2.48 & 3.48 & 3.89 \\
\hline SHP & 3.15 & 3.48 & 4.15 & 3.14 & 4.12 & 3.58 & 5.24 & 3.56 & 4.26 & 4.35 & 4.44 & 4.36 & 4.36 & 4.32 & 3.58 & 4.68 & 4.12 & 4.36 \\
\hline ICP & 3.12 & 3.47 & 5.33 & 2.85 & 3.14 & 3.19 & 3.11 & 4.98 & 3.45 & 3.12 & 5.64 & 3.1 & 3.14 & 3.26 & 3.54 & 3.68 & 3.49 & 2.98 \\
\hline BHS & 3.24 & 3.47 & 3.26 & 5.1 & 3.47 & 3.26 & 3.48 & 3.69 & 4.02 & 3.95 & 3.17 & 3.69 & 6.33 & 4.36 & 3.59 & 3.47 & 3.24 & 5.13 \\
\hline B-155-B & 2.85 & 4.35 & 3.15 & 5.14 & 3.96 & 4.12 & 5.01 & 4.26 & 3.14 & 4.23 & 4.12 & 4.26 & 4.21 & 4.11 & 4.29 & 4.36 & 4.16 & 4.1 \\
\hline B-127 & 4.32 & 4.58 & 3.14 & 2.96 & 3.41 & 3.12 & 3.25 & 3.56 & 4.12 & 4.21 & 4.26 & 4.23 & 4.1 & 4.06 & 3.48 & 3.51 & 3.11 & 3.19 \\
\hline B-192-5 & 3.68 & 3.48 & 3.25 & 2.87 & 3.14 & 3.45 & 2.96 & 2.47 & 3.89 & 3.47 & 4.1 & 3.11 & 2.58 & 2.47 & 3.14 & 2.96 & 3.15 & 3.17 \\
\hline B-127N-C & 4.29 & 4.21 & 3.41 & 5.12 & 6.21 & 4.56 & 6.24 & 4.35 & 4.68 & 5.12 & 4.68 & 4.89 & 3.48 & 4.26 & 4.69 & 4.59 & 4.65 & 4.69 \\
\hline B-48 & 2.45 & 3.14 & 2.69 & 2.74 & 2.86 & 3.14 & 3.26 & 3.1 & 5.14 & 3.69 & 3.25 & 3.41 & 3.74 & 3.36 & 3.14 & 3.41 & 3.15 & 3.57 \\
\hline GS-OSN-2004/1 & 3.68 & 2.98 & 2.45 & 3.01 & 3.45 & 4.13 & 3.68 & 3.89 & 3.48 & 2.69 & 2.75 & 3.12 & 2.63 & 4.23 & 3.15 & 2.98 & 3.11 & 4.12 \\
\hline GK-28/42 & 3.31 & 3.12 & 2.81 & 3.69 & 4.12 & 3.15 & 3.96 & 4.12 & 3.58 & 4.16 & 5.03 & 3.26 & 3.65 & 3.16 & 3.75 & 3.45 & 4.33 & 3.42 \\
\hline ТPР-ТCPP & 2.14 & 2.69 & 2.75 & 2.81 & 2.36 & 2.48 & 2.17 & 2.36 & 3.16 & 2.65 & 2.45 & 3.18 & 2.69 & 2.74 & 2.69 & 2.69 & 3.15 & 3.59 \\
\hline MHRF & 5.12 & 5.01 & 4.25 & 4.36 & 3.48 & 3.16 & 4.69 & 3.54 & 4.02 & 3.69 & 3.48 & 3.49 & & & & & & \\
\hline S-BASIN & 3.19 & 2.98 & 3.14 & 2.98 & & & & & & & & & & & & & & \\
\hline SATELLITE & 3.15 & 3.89 & 3.48 & 3.59 & 3.11 & 3.69 & 2.48 & 3.62 & & & & & & & & & & \\
\hline
\end{tabular}



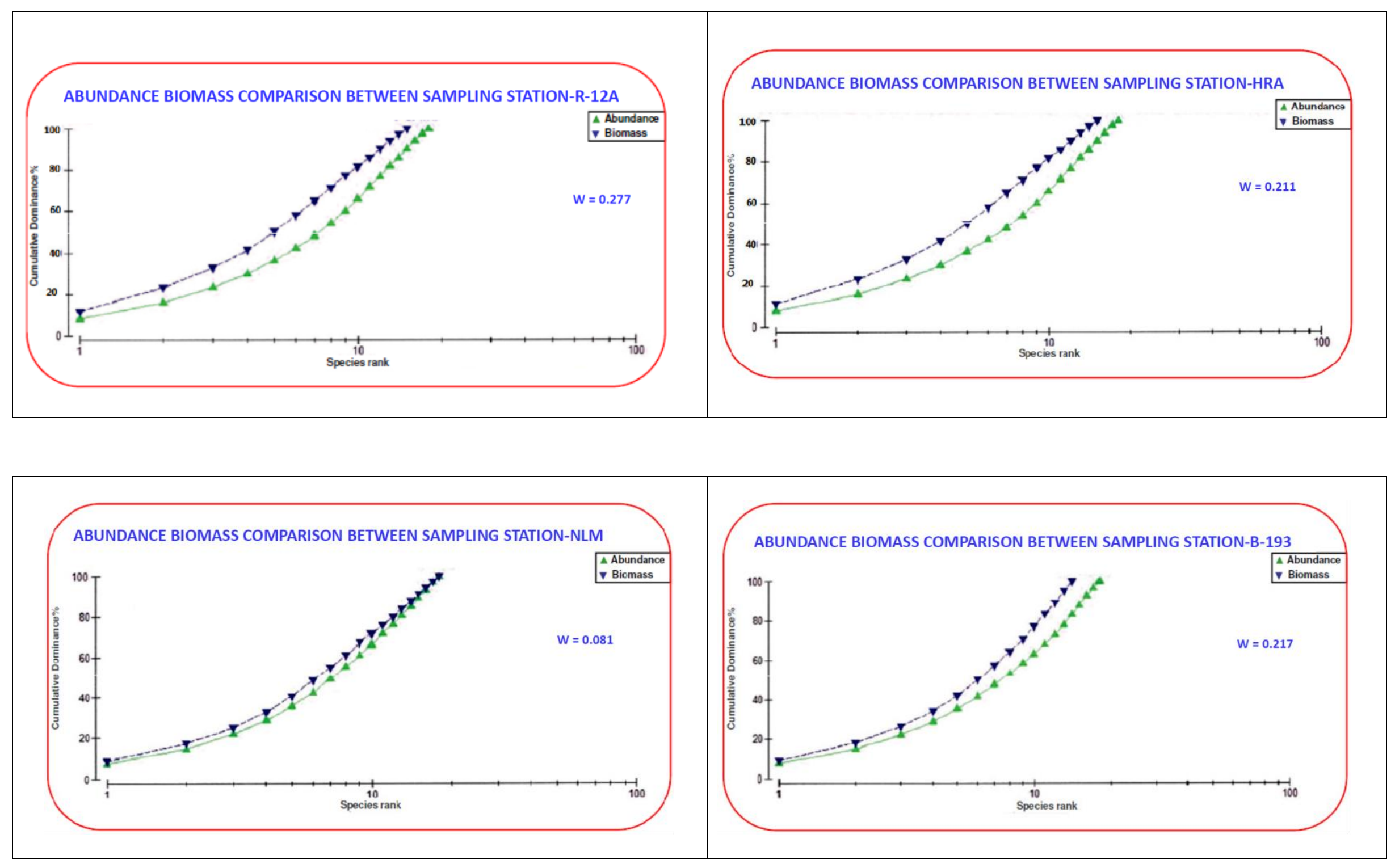

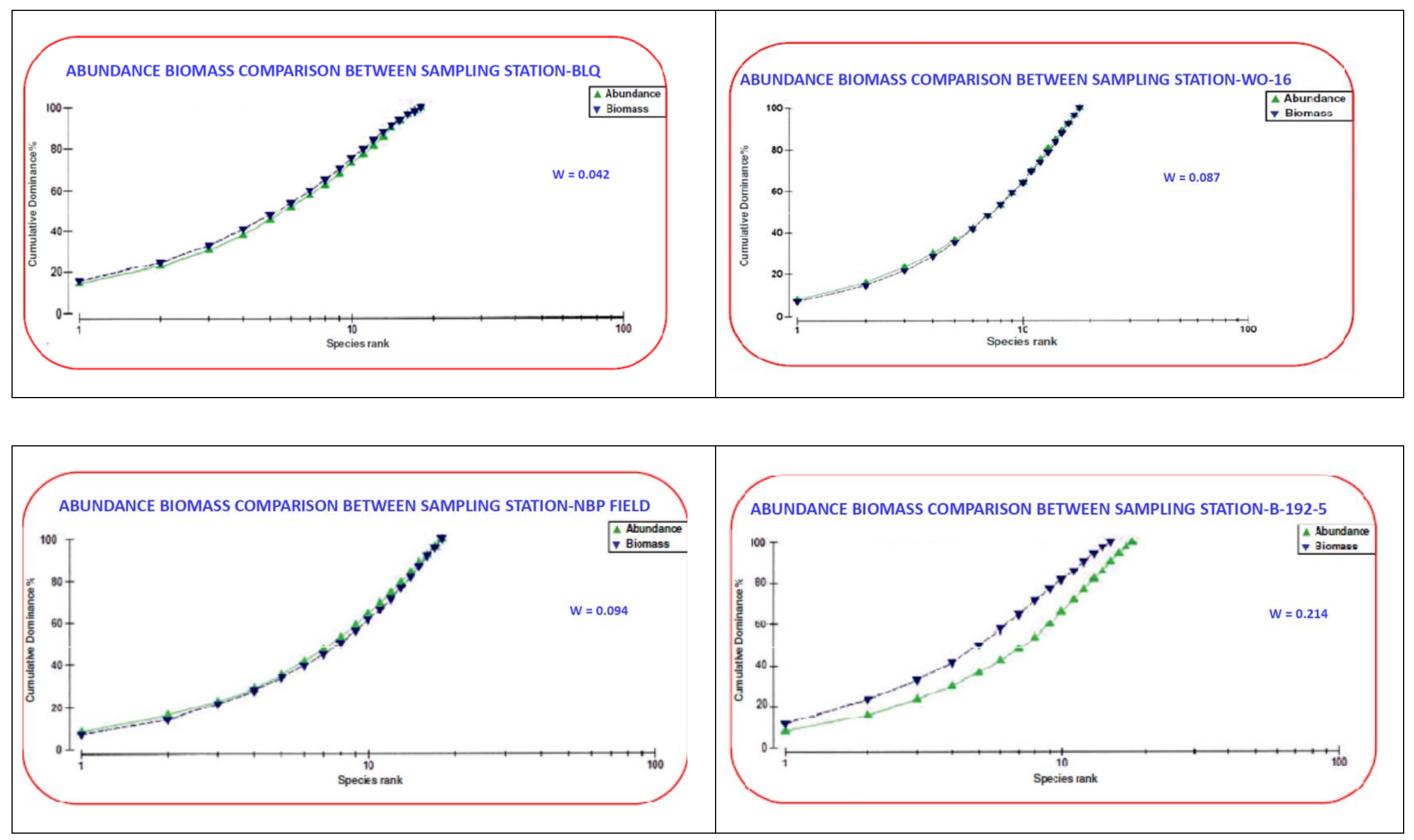

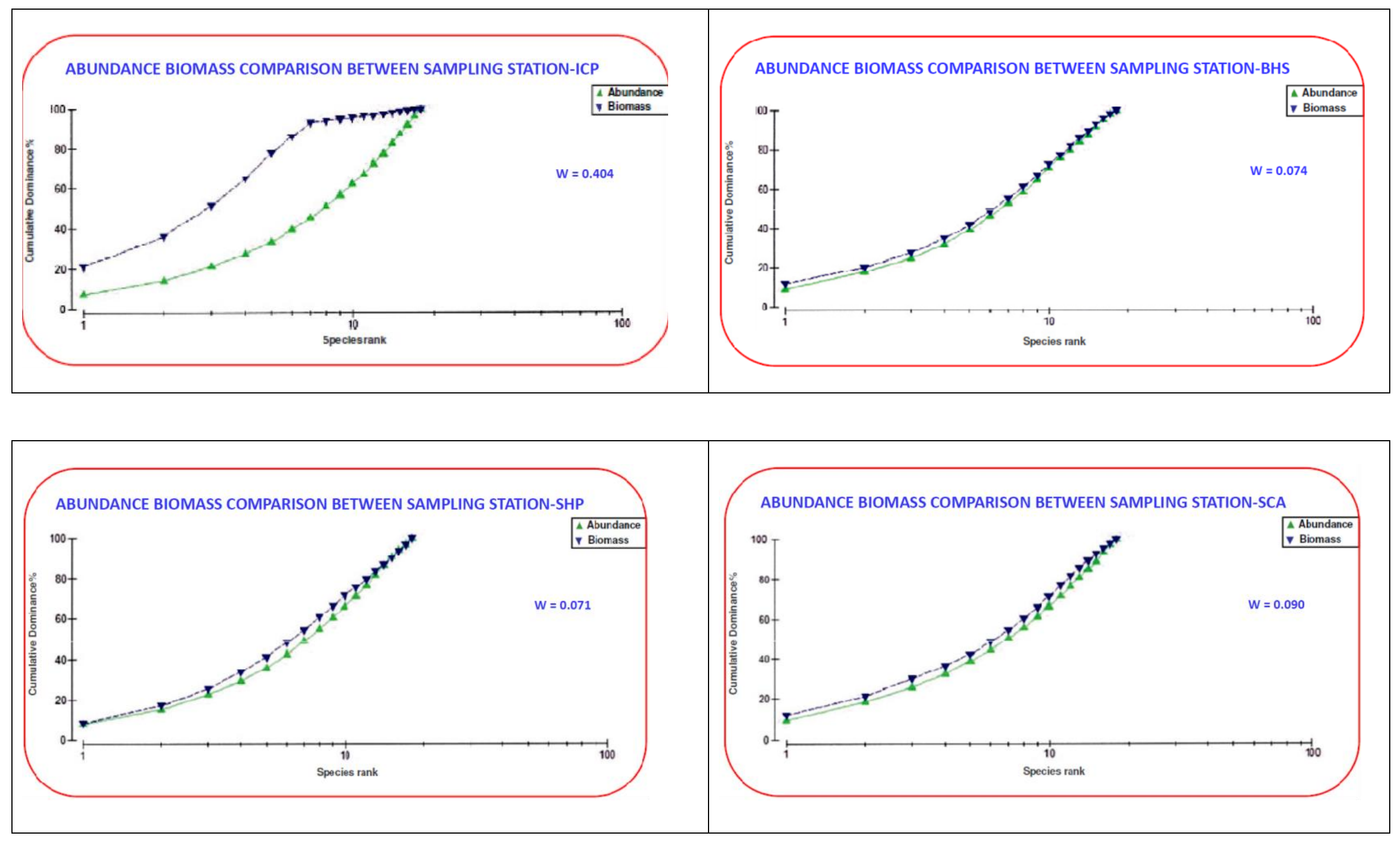

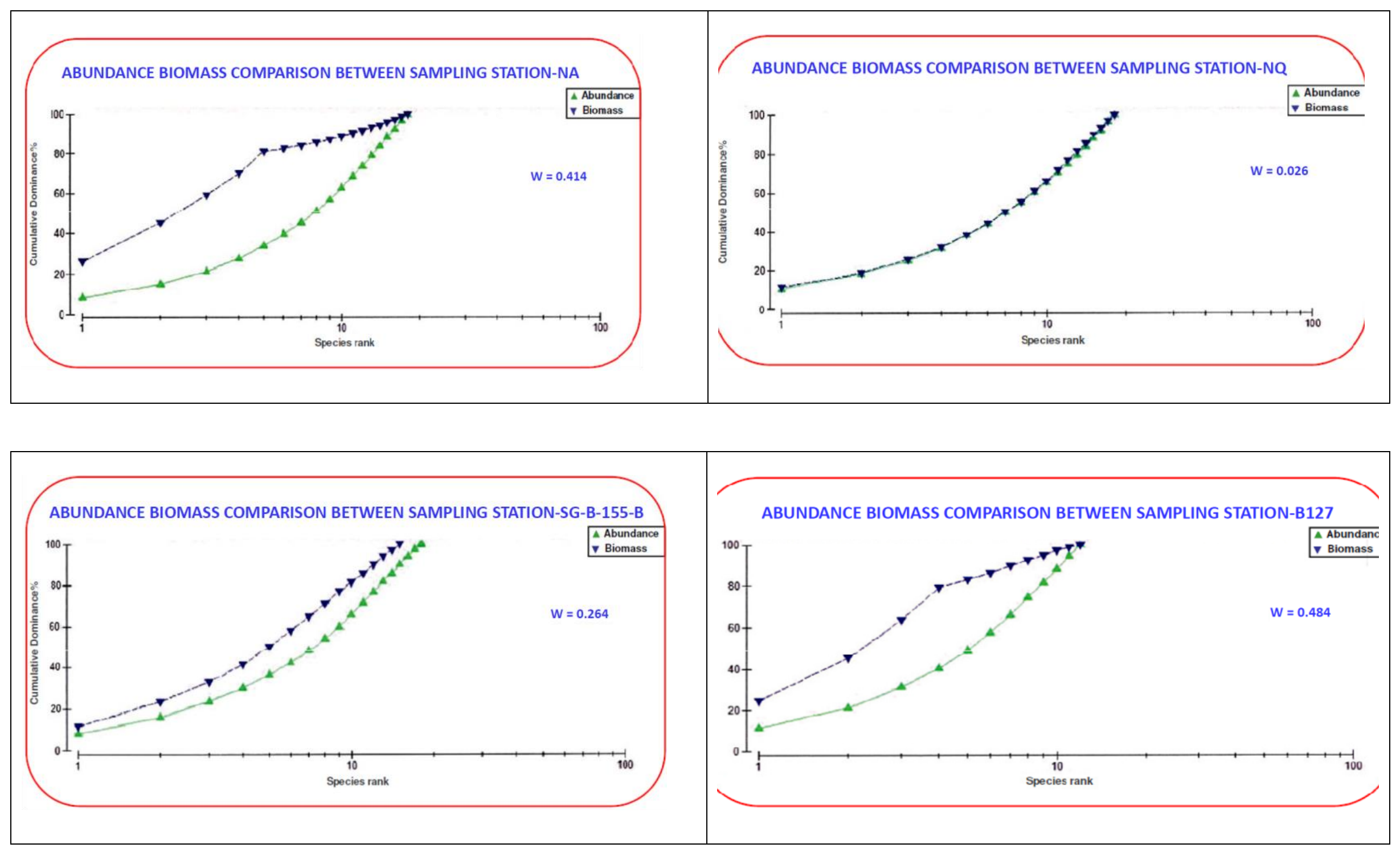

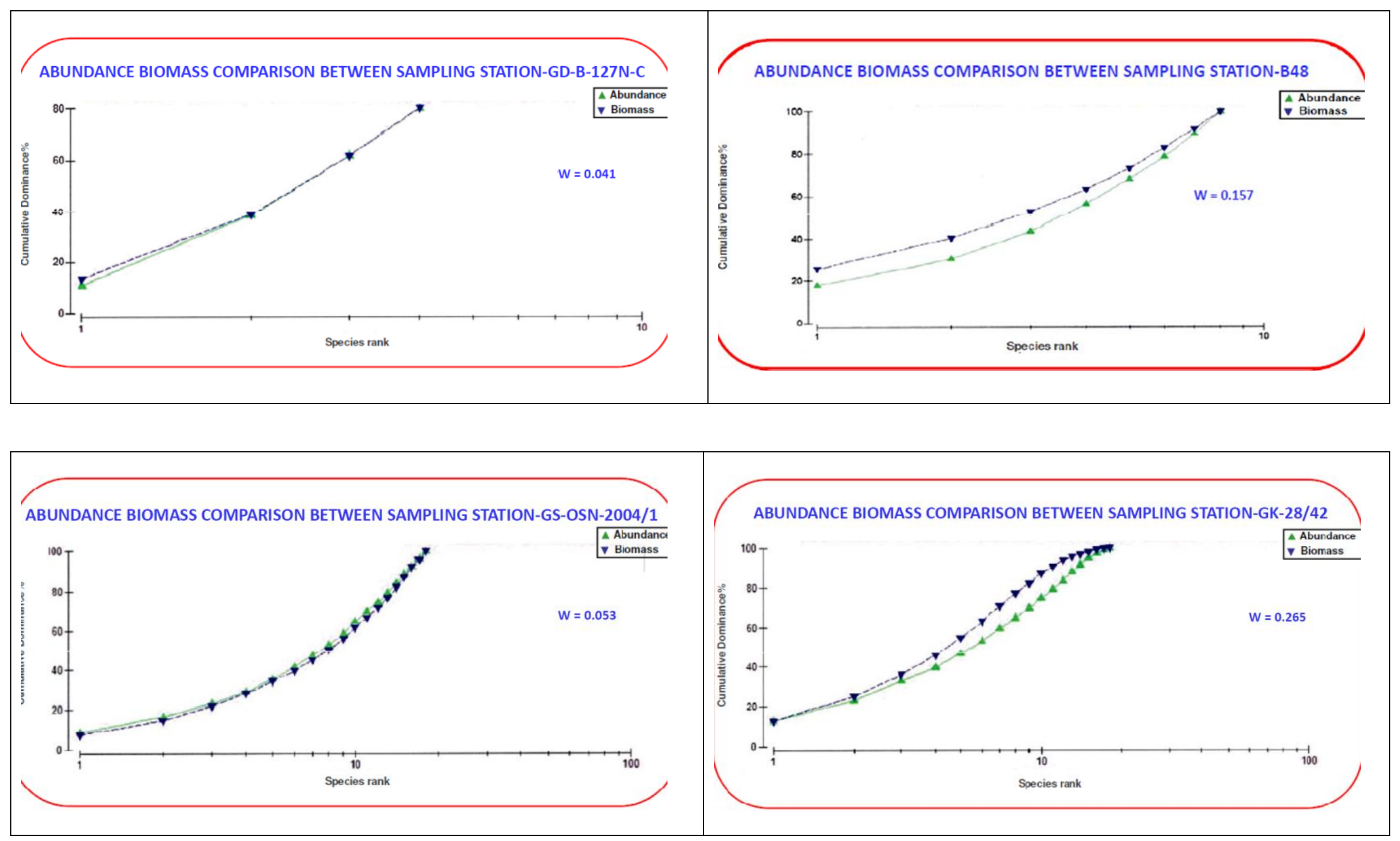


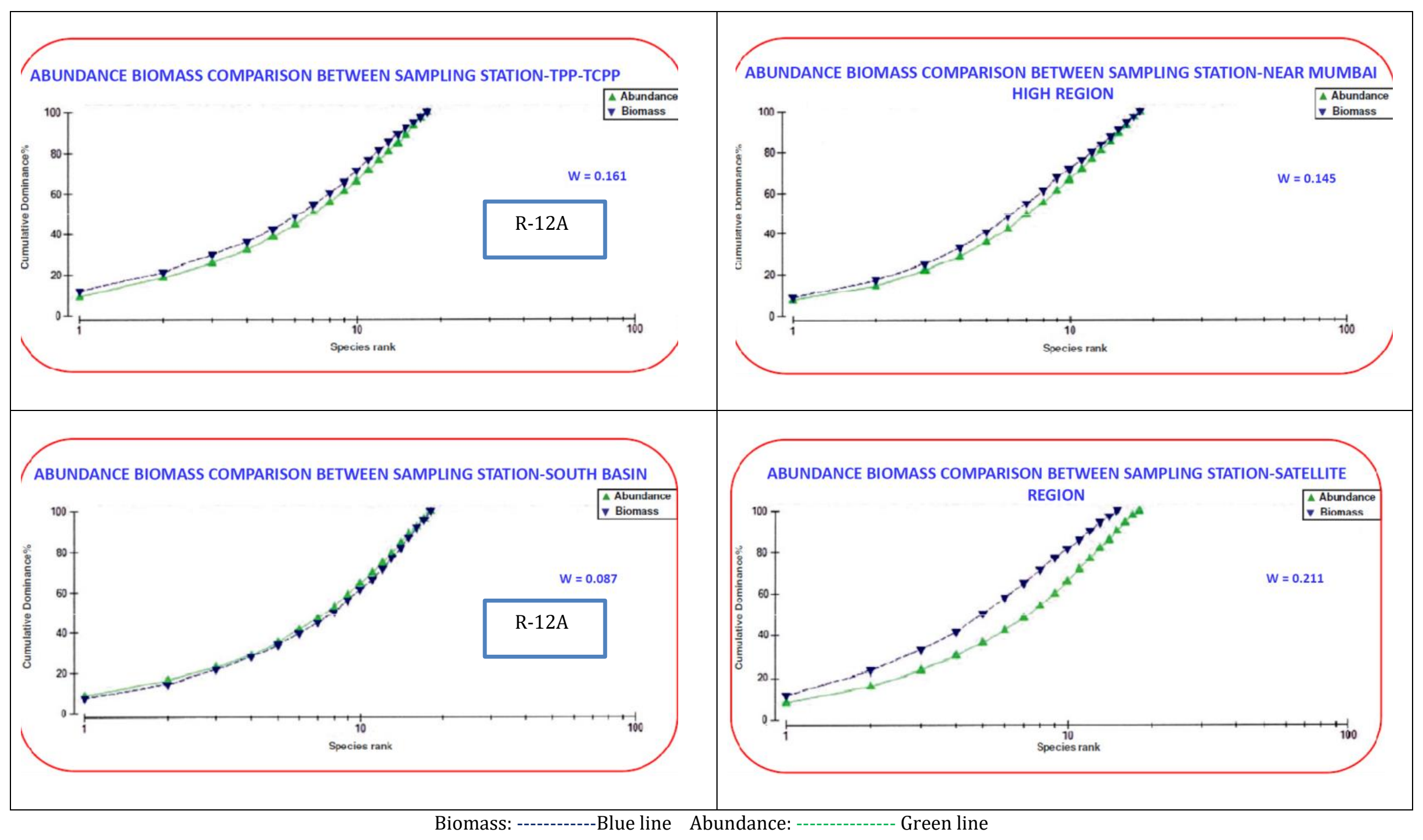


The biomass curve of ICP, NA, B-127, R-12A, HRA, B-193, B-192-5, B-155-B, GK-28/42 and Satellite Reference point dominates the abundance curve which signifies the areas around the these installations undisturbed. The benthic stress around these installations are minimum. At the same time, the areas of Satellite reference, which are considered as no influenced areas from E\&P activities, are found undisturbed.

Comparing the ABC curve around the installations NLM, BLQ, NQ, SCA, SHP, BHS, B 127N-C, GK-OSN-2204/1, WO-16, NBP filed, TPP-TCPP and Mumbai high \& South basin Reference point, it has been observed that, both the biomass and abundance curves come closer to each other. It signifies that the areas around the installations are moderately disturbed. The benthic community around those areas are under moderate stress.

\section{Conclusion}

- From the abundance / Biomass comparison $(\mathrm{ABC})$ method it can be concluded that few sampled installations (ICP, NA, B-127, R-12A, HRA, B-193, B-192-5, B-155-B, GK-28/42 and Satellite Reference point) are undisturbed. Other installations are moderately disturbed due to the ongoing activities, NLM, BLQ, NQ, SCA, SHP, BHS, B-127N-C, GK-OSN-2204/1, WO-16, NBP filed, TPP-TCPP and Mumbai high \& South basin Reference point) like E\&P activities, boat traffics and other fishing activities.

- Comparing the ABC analysis result of Mumbai High, South basin, \& Satellite reference points with those around ONGC installations, minor influence of operational activities of ONGC on few installations are observed on benthic fauna.

- As the paper is aimed to generate a valuable data bank on the environmental status of marine sediments and bottom-dwelling biological communities, it will help in taking care of marine environment during E\&P and other activities around western offshore areas. However, more modelling and comparative work is needed to establish these $\mathrm{ABC}$ analysis and their application in an ecosystem approach in these installations. The study will definitely open widows for further environment correlation studies

\section{Compliance with ethical standards}

\section{Acknowledgments}

The authors are grateful to the ONGC's management for encouraging for preparation and presentation of this paper. They also wish to acknowledge Shri Atul Garg, ED-HoI, IPSHEM for his continuous guidance and support.

Authors are also honestly grateful to Shri R K Srivastava, Director (Exploration), ONGC, for his encouragement for preparation of this paper.

\section{Disclosure of conflict of interest}

The authors declare no conflict of interest.

\section{References}

[1] Warwick RM. A new method for detecting pollution effects on marine macro benthic communities. Marine Biology. 1986; 92: 557-562.

[2] WARWICK RM, CLARKE KR. Relearning the ABC: taxonomic changes and abundance/biomass relationships in disturbed benthic communities. Mar Biol. 1994; 118: 739-744.

[3] OSPAR Guideline. Guidelines for monitoring methods to be used in the vicinity of European Seas. 1998.

[4] OEM report around ONGC installations in Western Offshore Region during. 2020-21.

[5] Marine pollution bulletin. 